\title{
Aplicaciones sensibles al contexto. Tendencias actuales.
}

\section{(Current trends in context-aware applications.)}

\author{
Andrea Loayza ${ }^{1}$, Rodrigo Proaño ${ }^{1}$, Diego Ordóñez Camacho ${ }^{1}$
}

\begin{abstract}
Resumen:
Las aplicaciones sensibles al contexto adaptan automáticamente su comportamiento y configuración, dependiendo de las condiciones del entorno y de las preferencias del usuario. Esta revisión del estado del arte presenta las tendencias en cuanto a técnicas y herramientas para el desarrollo de estas aplicaciones, así como los ámbitos de interés actual de la comunidad científica en esta área, donde se destaca la investigación en interfaces multimodales, localización, detección de actividades, control de interrupciones, aplicaciones predictivas y de ayuda al bienestar del usuario.
\end{abstract}

Palabras clave: aplicaciones sensibles al contexto; dispositivos móviles; diseño y desarrollo de software

\begin{abstract}
:
Context-aware applications adapt their behavior and settings according to the environment conditions and to the user preferences. This state-of-the-art survey identifies the current trends related to the technics and tools for the development of this kind of software, as well as the areas of interest of the scientific community on the subject. It stands out the research on multimodal interfaces, localization, activity detection, interruptions control, predictive and wellbeing applications.
\end{abstract}

Keywords: context-aware applications; mobile devices; software design and development

\section{Introducción}

Las aplicaciones sensibles al contexto son aquellas con la capacidad de adaptar el conjunto de actividades que ejecutan o conductas que presentan, dependiendo de las circunstancias físicas o situacionales en las que operan, sin que para ello deba mediar, o lo menos posible, la intervención del usuario. Idealmente deberían "desvanecerse en el background, para que el foco central sea el usuario y sus tareas, más que los dispositivos o las consideraciones técnicas" (Baldauf, Dustdar, \& Rosenberg, 2007, p. 1).

Este tipo específico de aplicaciones confía en la definición de contexto, el cual es un término utilizado en varias áreas y circunstancias, por lo cual sus definiciones pueden ser muy amplias. En

1 Universidad Tecnológica Equinoccial, Facultad de Ciencias de la Ingeniería, Quito - Ecuador (dordonez@ute.edu.ec)

Reconocimientos:

El presente trabajo ha sido realizado en el contexto del proyecto de investigación DADMDV- VI.UIO.ING.23, financiado y apoyado por el Instituto de Transferencia de Tecnologías, ITT, de la Universidad Tecnológica Equinoccial, Quito - Ecuador. 
el campo de trabajo de este estudio, orientado al desarrollo de aplicaciones, se aventura definir el contexto como el conjunto de objetos y características del entorno que al ser percibidas por elementos sensoriales, y puestas en relación con la situación particular de una entidad, dan lugar a una identidad claramente definible y reproducible. Los dos componentes principales en la definición son los elementos perceptibles que componen el entorno, y la entidad, o más específicamente en este caso el usuario, con las características que en el momento lo definen. La unión de estos dos componentes da lugar a esta identidad formada de manera no aleatoria, de la cual es posible extraer un patrón genérico que pueda aprovecharse por las aplicaciones sensibles al contexto.

Desarrollar aplicaciones sensibles al contexto requiere combinar activamente el conjunto de sensores del dispositivo con las preferencias del usuario respecto a sus posibles interacciones con dicho dispositivo y las actividades que se encuentra realizando, las cuales muy posiblemente estarán relacionadas a su localización. Hacer trabajar todos estos elementos de manera armoniosa requiere el uso de técnicas y metodologías específicas de software para facilitar el desarrollo de estas aplicaciones.

El objetivo de este estudio es en primer lugar presentar una recopilación de técnicas, y herramientas diseñadas específicamente para facilitar el desarrollo de aplicaciones sensibles al contexto (Sección 2), y en segundo lugar presentar la investigación actual orientada al desarrollo de estas aplicaciones, distinguiendo los principales ámbitos de interés (Sección 3). Finalmente se establecen conclusiones sobre el estado del arte y se proporcionan recomendaciones de trabajo a futuro (Sección 4).

\section{Diseño y desarrollo de aplicaciones}

Esta sección se enfoca en revisar técnicas, métodos, herramientas y librerías concebidas específicamente para el desarrollo de aplicaciones sensibles al contexto. En primer lugar se presentan estudios que recopilan técnicas y metodologías orientadas al diseño y modelado de aplicaciones, al manejo de información en proveniencia de los sensores, y al prototipado y evaluación de aplicaciones. Luego se revisa un grupo representativo de herramientas y librerías de soporte, y finalmente se presenta un grupo de investigaciones orientadas al problema particularmente relevante de las interfaces multimodales.

\subsection{Técnicas y metodologías}

Baldauf et al., (2007) presentan en primer lugar una perspectiva histórica sobre el interés en el desarrollo de sistemas sensibles al contexto, que recoge información desde 1991 . Luego presentan diferentes principios de diseños y modelos de contexto utilizados en varios sistemas, concentrándose especialmente en soluciones orientadas al middleware y basadas en servidores. Comparan estos sistemas revisando sus distintos enfoques, sus diferencias y similitudes, y los 
discuten poniendo de relieve sus ventajas y desventajas. Finalmente destacan la eficacia de las arquitecturas que aplican la división por capas entre el hallazgo y reconocimiento de recursos, la adquisición de información de contexto a través de los sensores, la construcción y administración de los modelos, el procesamiento y toma de decisiones, y la interfaz de presentación. Dependiendo del modelo utilizado y de la orientación de la aplicación se recomienda dos capas adicionales, una para el manejo de información histórica, y otra para la seguridad y privacidad de la información y los recursos. Para este último tema, la seguridad, son de gran utilidad los tratados de Enck, Octeau, McDaniel y Chaudhuri (2011), Rai (2013), Gunasekera (2012), Misra y Dubey (2013), orientados a la plataforma Android. Para el caso de iOS se puede consultar a Miller et.al. (2012) y Zdziarski (2012) y como tratado general es recomendable revisar a Androulidakis (2012).

Considerando que los sistemas sensibles al contexto son difíciles de diseñar, desarrollar y mantener, Wei y Chan (2007) proporcionan una revisión que se enfoca en el principio de que para atacar las dificultades inherentes al problema, las aplicaciones sensibles al contexto deben considerar cuatro aspectos fundamentales: qué es el contexto, cómo percibirlo, cómo representarlo y cómo adaptarse al mismo. En esta línea es de particular interés el tratado de Milette y Stroud (2012) sobre cómo utilizar, en el sistema Android, los sensores del dispositivo para generar aplicaciones que interactúen con el usuario y el entorno de manera efectiva. Para iOS se puede consultar a Allan (2011).

La dificultad que presenta el trabajo con estos sistemas, especialmente cuando se añade a la ecuación el uso de dispositivos móviles que hacen uso de estas tecnologías, obliga a que en el desarrollo de estas aplicaciones sea de especial interés el uso de técnicas específicas de prototipado y evaluación orientadas a los sistemas sensibles al contexto. De Sá y Carriço (2011) estudian este problema, enfocándose sobre todo en la interacción con el entorno y las interfaces de usuario, de las aplicaciones móviles sensibles al contexto. Una interesante conclusión de este trabajo es que tanto los prototipos como las técnicas de evaluación deben ser diseñados para utilizarse y probarse fuera del laboratorio, en condiciones reales de uso.

\subsection{Herramientas y librerías}

El desarrollo de aplicaciones sensibles al contexto necesita considerar un conjunto diferente de sensores, eventos y acciones que en el caso de aplicaciones clásicas orientadas al negocio. Estos elementos requieren la implementación de ciertos patrones específicos en el código, los cuales incrementan la complejidad al programar este tipo de sistemas.

Una alternativa para reducir esta complejidad es la que se propone con JCOp (Schuster, Appeltauer, \& Hirschfeld, 2011), el cual es un lenguaje de programación orientado al contexto que facilita esta tarea al implementar estos patrones mediante la inclusión en el lenguaje de un set de 
reglas de adaptación que toman en cuenta información proveniente de los sensores, considerándola como eventos externos desencadenadores de acciones.

Du \& Wang (2008) apuntan a la programación automática de aplicaciones, y para este efecto proponen un framework específicamente orientado a la generación de aplicaciones sensibles al contexto. Este entorno permite usar un conjunto de especificaciones con las cuales se puede definir la conducta de las aplicaciones mediante la generación de reglas que pueden atarse a un conjunto de acciones dependientes del contexto. A partir de estas especificaciones el modelo del framework genera automáticamente la aplicación.

La generación de librerías específicas, orientadas al manejo de ciertas tareas sensible al contexto, que puedan ser incluidas en una aplicación para facilitar su desarrollo es también una preocupación en este campo. BeTelGeuse (Nurmi, Kukkonen, Lagerspetz, Suomela, \& Floréen, 2007) y TreasurePhone (Seifert, De Luca, Conradi, \& Hussmann, 2010) son dos ejemplos interesantes de esta línea de investigación.

BeTelGeuse permite recuperar información de contexto mediante la detección y conexión con dispositivos Bluetooth. Es una aplicación de soporte que facilita la construcción de aplicaciones más específicas que la utilicen como soporte en el proceso de hallazgo y reconocimiento de recursos.

TreasurePhone ofrece un esquema de seguridad dependiente del contexto, donde el usuario define diferentes esferas, cada una de ellas atadas a diferentes características de contexto, y con diferentes condiciones de seguridad. El cambio de esfera de seguridad se hace automáticamente al cambiar de contexto.

\subsection{Interfaces multimodales}

La interacción del usuario con los dispositivos móviles sensibles al contexto es también un área activa de investigación. Es particularmente interesante el estudio orientado hacia el uso de interfaces multimodales, las cuales se adapten dinámicamente al contexto para ofrecer al usuario una mejor experiencia de uso sin necesitar recurrir a configuraciones manuales. Más allá, diferentes tipos de usuarios pueden requerir diferentes tipos de interfaces en contextos similares.

Khalil \& Connelly (2005a) estudian las reacciones de los usuarios de dispositivos móviles frente a dos posibilidades: configuración puramente automática para adaptarse al contexto, 0 configuración manual. El estudio arroja como resultado que la configuración automática es preferida sobre la manual, sin embargo también sugiere que un enfoque híbrido, automáticomanual, es preferido sobre aquel puramente automático, lo cual podría explicarse por el gusto que el usuario experimenta al manipular su teléfono. 
Lemmelä, Vetek, Mäkelä, \& Trendafilov (2008) se interesan en evaluar las mejores estrategias para el diseño de interfaces multimodales de envío de mensajes en dos situaciones: caminando y conduciendo. Entre los resultados del estudio se encontró que si bien al conducir se prefiere la interacción por comandos vocales, esta no se considera útil al caminar, situación en la que se prefiere el tacto y los gestos moviendo el teléfono.

La ayuda que diferentes tipos de interfaces puedan brindar a las personas de edad es objeto del estudio de Naumann, Wechsung, \& Hurtienne (2010). Ellos muestran que las personas de edad prefieren el uso de interfaces multimodales, con las cuales pueden interactuar de mejor manera al cambiar de acuerdo al contexto. En segundo lugar destacan que en este grupo las interfaces táctiles son las preferidas, y que aquellas con la menor acogida son las de control mediante el movimiento del dispositivo.

\section{3. Ámbitos de interés}

Amplias son las posibilidades de interacción entre usuarios y entorno, a través de las aplicaciones sensibles al contexto. Esta misma amplitud, sin embargo, obliga a delimitar tanto la investigación y desarrollo de aplicaciones orientadas al contexto, como el presente estudio del estado del arte. La revisión de las tendencias actuales realizada nos presenta sobre todo dos campos de acción a los que la comunidad científica presta especial atención. En primer lugar destaca el problema de la localización exacta del usuario con miras a aprovechar diversos tipos de información de relevancia para el mismo. En segundo lugar el interés se dirige a la detección de las actividades que realiza el usuario, en conjunto con la búsqueda de configuraciones específicas de control de interrupciones molestas por parte del dispositivo. Algunas variantes en este punto son las aplicaciones predictivas y aquellas que buscan mejorar la calidad de vida del usuario.

\subsection{Localización}

Uno de los mayores intereses en el área de las aplicaciones sensibles al contexto es conseguir una ubicación precisa del usuario. Para localizar una persona en el entorno, el sensor de mayor uso es, sin lugar a dudas, el GPS. Sin embargo el GPS no es suficientemente preciso en situaciones donde la aglomeración de alternativas es muy alta, o bajo techo, como sería el caso de por ejemplo los centros comerciales. Varios estudios intentan combinar distintos tipos de sensores para conseguir la precisión requerida y utilizar esta información en diferentes actividades.

CAMM (Freyne et al., 2007) es un sistema que además del GPS se vale del sensor Bluetooth como sensores de contexto, para detectar la ubicación de una persona en el entorno, o inclusive su cercanía con otras personas. Este sistema está orientado al envío de mensajes personalizados y demuestra su relevancia especialmente al poderse utilizar para enviar recordatorios en el 
momento preciso, por ejemplo cuando la persona entra a un salón, o cuando se detecta que se encuentra con otra persona, dentro de un rango de tiempo determinado.

SerPens (Bhattacharya, Kukkonen, Nurmi, \& Floréen, 2008) complementa la información proveniente del GPS, sirviéndose también de las antenas WiFi. A la información de ubicación resultante le añade información semántica del contexto, la cual comparte con los demás usuarios de la red valiéndose para ello de Bluetooth, mediante la librería BeTelGeuse (Nurmi et al., 2007).

Ofstad, Nicholas, Szcodronski, y Choudhury (2008), en cambio estudian cómo mejorar la información de localización en proveniencia de GPS, mediante el uso de información proveniente del acelerómetro. El estudio demuestra que esta técnica puede ser útil para corregir errores de localización en los mapas, permitiendo un uso más acertado, por ejemplo al enviar publicidad sobre el lugar donde se encuentra la persona.

SurroundSense (Azizyan \& Choudhury, 2009) se basa en la hipótesis de que diferentes entornos tienen una firma foto-acústica específica. De esta manera trata de resolver las limitaciones de los sistemas de localización mediante GPS o WiFi, mediante el uso de sensores de sonido y luz que capturen esta identidad del entorno y la utilicen para localizar la posición con mayor exactitud.

Para superar las inexactitudes de la localización por GPS, Bicocchi, Castelli, Mamei, y Zambonelli (2011) utilizan información de las actividades que realiza el usuario, la cual se pone en relación con la ubicación gracias a una base de datos externa donde estas relaciones de sentido común, como las llaman, se guardan. El estudio demuestra un alto incremento en la exactitud de la localización

Leung, Lee, y Lee (2011), toman un enfoque colaborativo, y recogen información proporcionada por los usarios de la red, considerando actividades, preferencias temporales, diferentes clases de usuarios. Mediante esta información ajustan no solo la localización, sino que personalizan y especifican más las recomendaciones que se entregan al usuario.

La localización correcta es particularmente importante para las aplicaciones orientadas al turismo y a guiar a una persona en un entorno específico. Varias investigaciones existen sobre este tipo de aplicaciones, las cuales utilizan el contexto para este efecto. Algunos ejemplo relevantes son MoCCha (Westermann \& Möller, 2012), que brinda información variada a estudiantes y visitantes en un campus universitario. Yu y Chang (2009) añaden a la localización del usuario información de sus preferencias personales para proporcionar recomendaciones turísticas basadas en la localización del usuario, tomando en cuenta sus preferencias personales. Más recientemente Emmanouilidis, Koutsiamanis, y Tasidou (2013) presentan una revisión de técnicas para aplicaciones móviles que dan información del lugar, guiando a la persona por ejemplo en museos o instituciones públicas. 


\subsection{Detección de actividades y control de interrupciones}

La ubicuidad de los dispositivos móviles, constantemente en línea, hace posible que se reciba información, ya sean emails, mensajes de texto o llamadas en cualquier lugar y momento. La llegada de estos mensajes viene acompañada de un anuncio generalmente sonoro, aunque también puede ser por medio de la vibración del dispositivo. Este último, aunque menos invasivo, puede ser un elemento distractor en circunstancias donde la total atención del usuario debe enfocarse en ciertas actividades. Si bien los dispositivos móviles cuentan en su gran mayoría con opciones para desactivar los avisos, muchas veces el usuario olvida utilizarlas, lo que puede generar molestias a quienes se encuentran en las cercanías y al mismo usuario.

Un campo activo de investigación, dentro del área de los sistemas sensibles al contexto, es buscar los mecanismos adecuados para automáticamente detectar aquellas circunstancias en que el usuario debería o no ser avisado. A continuación presentamos algunos trabajos representativos.

Uno de los pioneros en esta línea es SenSay (Siewiorek et al., 2003) que hace uso de un conjunto de sensores para luz, movimiento y sonido, distribuidos alrededor del cuerpo del usuario. La información recibida por los sensores durante los últimos minutos se analiza y sopesa para evitar bruscos y muy frecuentes cambios de estado. Finalmente el sistema puede tomar la decisión de cambiar a uno de cuatro estados posibles: no interrumpir, en espera, activo, y por defecto. Cada uno de estos estados implementa una serie de acciones predefinidas como por ejemplo deshabilitar el timbre y activar el vibrador. Adicionalmente cuenta con la capacidad de avisar a quien llama del estado actual del usuario.

Ho e Intille (2005) exploran las posibilidades de utilizar los dispositivos móviles para enviar información al usuario de manera proactiva, al mismo tiempo minimizando las interrupciones mientras realiza otro tipo de tareas. Mediante el uso de un dispositivo con varios acelerómetros para detectar el desplazamiento y el cambio de postura, se midió de manera experimental la receptividad del usuario a los mensajes enviados en las transacciones de una actividad a otra, comparado a mensajes enviados a momentos aleatorios. Se comprobó que los mensajes recibidos entre actividades eran mejor recibidos al no causar, entre otras cosas, sobrecarga de información.

CASIS (Leong, Kobayashi, Koshizuka, \& Sakamura, 2005) utiliza un sistema de reconocimiento vocal para controlar el dispositivo, y está diseñado para operar en un ambiente inteligente, recibiendo información de contexto de los sensores del entorno, así como de los sensores en el dispositivo. La información recibida del entorno y del dispositivo se combina con el sistema de reconocimiento vocal utilizando una red Bayesiana. Se reporta un $41 \%$ de reducción de errores en la configuración, si se compara con un sistema que no aprovechase la información de contexto. Springer, Wustmann, Braun, Dargie y Berge (2008) también estudian el proceso de captura del 
contexto, abstracción y toma de decisiones en un escenario inteligente, tomando como caso de estudio una sala de reuniones.

Un sistema de navegación para peatones es propuesto por Yamabe, Takahashi y Nakajima (2008), el cual busca disminuir la sobrecarga sensorial a la que se somete al usuario, efectivizando la información enviada. El problema de sobrecarga sensorial se complica mientras el usuario se encuentra en movimiento ya que reduce la atención que presta al dispositivo. Esto obliga a que la información de importancia llegue al usuario incluso con la mínima atención de su parte.

Driver \& Clarke (2008) utilizan la información del contexto para superar las limitaciones de las agendas estáticas tradicionales. La aplicación propuesta reordena dinámicamente la agenda, basándose en la información de contexto que va emergiendo.

CAMeL, context-aware mobile learning, es un prototipo para investigar cómo aprovechar el contexto para proponer información y actividades de aprendizaje específicas y orientadas al momento y entorno mediante el dispositivo móvil (Thüs et al., 2012).

Figo, Diniz, Ferreira y Cardoso (2010) investigan diferentes maneras de conocer la actividad que está realizando el usuario, por ejemplo caminando, corriendo o sentado, mediante el uso del acelerómetro. Esta información se asocia a información de localización proporcionada por el GPS para discriminar de manera más precisa la actividad en particular que el usuario realiza en ese momento.

La aplicación de Ketabdar y Yüksel (2010)utiliza la información de los movimientos que está realizando el usuario, junto al análisis del sonido ambiental, para así distinguir diversos contextos en los que el usuario se puede encontrar, y modificar la alerta de llamadas (timbre, vibrador $u$ otros) acorde a la situación.

El estar siempre conectado a las redes sociales puede generar considerables niveles de estrés en muchos usuarios. Sambasivan, Ventä, Mäntyjärvi, Isomursu y Häkkilä (2009) estudian los problemas que puede provocar esta conexión permanente y cómo, mediante aplicaciones sensibles al contexto, reducir la intrusión e inclusive el estrés que esto genera, manteniendo al mismo tiempo un alto nivel de sociabilidad en las redes.

En Japón, el Human Activity Sensing Consortium (Kawaguchi et al., 2011) busca generar una gran base de datos con información de las actividades del ser humano, obtenida mediante acelerómetros. El objetivo final es apoyar a la nueva generación de computadores enfocados en su servicio directo en contacto con el ser humano.

Ouchi y Doi inicialmente proponen una aplicación que detecta en tiempo real la actividad que realiza la persona utilizando el acelerómetro, reportando un $95 \%$ de exactitud. Cuando la 
aplicación detecta que la condición es "trabajando", hace una clasificación adicional con el sensor acústico para sub clasificarla en otras siete categorías de trabajo, reportando un $85 \%$ de éxito en esta clasificación (2011). En una segunda etapa distinguen entre actividades bajo techo y externas mediante el uso de dos motores de detección diferente, los cuales se intercambian dependiendo de información tomada del GPS. Las actividades internas pueden ser descansando, caminando, aspirando, lavándose los dientes entre otras. Las actividades externas reconocen el tipo de movimiento: reposo, caminado, corriendo y en un vehículo (2012).

Se ha encontrado una tendencia a la investigación en aplicaciones que puedan de alguna manera mejorar la calidad de vida de las personas en aspectos como la salud, el deporte, el desplazamiento seguro, y otros.

ActiveSpeech (Everitt, Harada, Bilmes, \& Landay, 2007) es un entrenador deportivo electrónico que integra cuatro partes: un sistema de reconocimiento de etiquetas RFID instaladas en equipamiento de gimnasio, un sistema de reconocimiento vocal de acciones, y el sistema inteligente de entrenamiento. ActiveSpeech intenta que el usuario lleve registro de sus actividades en el gimnasio sin necesidad de interrumpir su ejercicio para manipular su registro. El usuario brinda comandos vocales indicando la actividad que realiza, y el sistema se ayuda de las etiquetas RFID, parte del contexto, para desambiguar las instrucciones al conocer el equipo con el que se trabaja.

En la misma línea, Pernek, Hummel y Kokol (2013) utilizan el acelerómetro de los dispositivos móviles para capturar información sobre el ejercicio que el usuario está realizando. Esta información se procesa y se brinda como retroalimentación al usuario para que pueda mejorar su entrenamiento de resistencia. Un ejemplo de esta información es indicar al usuario cuántas repeticiones, en una serie, han sido correctamente efectuadas.

El estudio realizado por Kimura, Ebisui, Funabashi, Yoshii y Nakajima (2011) intenta averiguar si los sensores instalados en un dispositivo móvil pueden ser suficientes como para poder generar aplicaciones persuasivas, donde se busca incitar o inhibir al usuario frente a ciertas conductas. Aplicaciones de este tipo existen, sin embargo utilizan hardware específico de difícil manipulación, que suele desanimar a nuevos, probables usuarios.

Una variación interesante a la detección de las actividades que se realizan en el momento presente, es la predicción de actividades sobre la base del contexto actual, sumado probablemente a información histórica recopilada por el sistema.

Khalil \& Connelly (2005b) estudian cómo la información en la agenda o calendario del usuario puede utilizarse para inferir sus actividades y automáticamente configurar el dispositivo de acuerdo a las mismas. El estudio muestra que la información de la agenda puede utilizarse con gran exactitud para predecir tanto las actividades del usuario como la configuración adecuada del 
dispositivo. Adicionalmente, la correlación entre actividades y configuración es alta cuando se considera cada usuario individualmente, sin embargo en el estudio no se encontró consistencia al tratar de establecer correlaciones entre diferentes usuarios.

El estudio realizado por Khan (2011) busca predecir los deseos del usuario con respecto a las llamadas, mensajes o mails entrantes, dependiendo del entorno en el que se encuentre. Para esto en primer lugar recopiló información tomada de los sensores de luz, temperatura y sonido. Luego hizo uso de algoritmos de machine learning, mediante los cuales reporta haber clasificado correctamente las diferentes instancias.

Miller (2013) revisa una conjunto de aplicaciones predictivas que anticipan lo que el usuario desea, como por ejemplo búsqueda predictiva y asistentes personales robotizados. En este tipo de aplicaciones, tales como Google Now, Cue, reQall, Donna, Tempo AI, MindMeld y Evernote, el contexto, ubicación, hora, día de la semana e historial de actividad se convierten en el patrón de búsqueda, el cual se ejecuta automáticamente, y de manera proactiva brinda resultados y recomendaciones.

Finalmente, EmotionSense (Sayid, 2013) va todavía más allá en el campo de la detección y de la predicción. EmotionSense realiza un seguimiento de llamadas y patrones en los mensajes de texto y trata de averiguar lo que la persona siente. La aplicación se apoya en preguntas efectuadas al usuario ocasionalmente para averiguar sus emociones en el momento. La información de contexto se captura mediante el uso de los sensores, y finalmente la aplicación vincula toda esta información para discriminar los picos emotivos mostrando cuándo el usuario se encuentra, por ejemplo, más estresado. Uno de los objetivos de esta aplicación es ayudar al usuario a mejor su estado de bienestar.

\section{Conclusiones y recomendaciones}

La presente revisión del estado del arte presenta en primer lugar diversas recomendaciones para abordar el desarrollo de aplicaciones sensibles al contexto. Se cubre la arquitectura, el manejo de sensores, y el prototipado y evaluación del sistema resultante. Se destacan las recomendaciones de arquitectura en capas, así como la realización de pruebas de campo. En lo relativo a elementos específicos dentro de las aplicaciones, predomina la investigación en técnicas de interfaces multimodales donde se revela la preferencia de los usuarios por enfoques híbridos que combinen configuraciones automática y manuales.

El segundo bloque comprendido en esta revisión concierne a la investigación y desarrollo de aplicaciones utilitarias, donde la atención de la comunidad científica se enfoca en la localización precisa del usuario para proporcionarle información relevante, y en la detección de las actividades con especial preocupación en el control de interrupciones. Conviene poner de relieve una tendencia hacia la generación de aplicaciones predictivas y de mejora del bienestar del usuario. 
Finalmente, la evidencia muestra una tendencia sostenida hacia el desarrollo de aplicaciones inteligentes sensibles al contexto. Las propuestas recopilan grandes cantidades de información y hacen uso de técnicas de machine learning para encontrar relaciones y extraer información significativa del contexto que sirva de aporte tanto para el desenvolvimiento del usuario en el entorno actual, como para adaptarse a situaciones futuras predecibles.

\section{Bibliografía}

Allan, A. (2011). Basic Sensors in iOS: Programming the Accelerometer, Gyroscope, and More (1st ed.). Sebastopol: O’Reilly Media.

Androulidakis, I. I. (2012). Mobile Phone Security and Forensics: A Practical Approach (1st ed.). Dordrecht: Springer.

Azizyan, M., \& Choudhury, R. R. (2009). SurroundSense: mobile phone localization using ambient sound and light. SIGMOBILE Mob. Comput. Commun. Rev., 13(1), 69-72. doi:10.1145/1558590.1558605

Baldauf, M., Dustdar, S., \& Rosenberg, F. (2007). A survey on context-aware systems. International Journal of Ad Hoc and Ubiquitous Computing, 2(4), 263. doi:10.1504/IJAHUC.2007.014070

Bhattacharya, S., Kukkonen, J., Nurmi, P., \& Floréen, P. (2008). SerPens: a tool for semantically enriched location information on personal devices. In Proceedings of the ICST 3rd international conference on Body area networks (pp. 30:1-30:2). ICST, Brussels, Belgium, Belgium: ICST (Institute for Computer Sciences, Social-Informatics and Telecommunications Engineering).

Retrieved from http://dl.acm.org/citation.cfm?id=1460257.1460297

Bicocchi, N., Castelli, G., Mamei, M., \& Zambonelli, F. (2011). Augmenting mobile localization with activities and common sense knowledge. In Proceedings of the Second international conference on Ambient Intelligence (pp. 72-81). Berlin, Heidelberg: Springer-Verlag. doi:10.1007/978-3-642-25167-2_8

De Sá, M., \& Carriço, L. (2011). Designing and Evaluating Mobile Interaction: Challenges and Trends. Found. Trends Hum.-Comput. Interact., 4(3), 175-243. doi:10.1561/1100000025 
Driver, C., \& Clarke, S. (2008). An application framework for mobile, context-aware trails. Pervasive Mob. Comput., 4(5), 719-736. doi:10.1016/j.pmcj.2008.04.009

Du, W., \& Wang, L. (2008). Context-aware application programming for mobile devices. In Proceedings of the $2008 C^{3} S^{2} E$ conference (pp. 215-227). New York, NY, USA: ACM. doi:10.1145/1370256.1370292

Emmanouilidis, C., Koutsiamanis, R.-A., \& Tasidou, A. (2013). Review: Mobile guides: Taxonomy of architectures, context awareness, technologies and applications. J. Netw. Comput. Appl., 36(1), 103-125. doi:10.1016/j.jnca.2012.04.007

Enck, W., Octeau, D., McDaniel, P., \& Chaudhuri, S. (2011). A Study of Android Application Security. In Proceedings of the 20th USENIX Conference on Security (pp. 21-21). Berkeley, CA, USA: USENIX Association. Retrieved from http://dl.acm.org/citation.cfm?id=2028067.2028088

Everitt, K. M., Harada, S., Bilmes, J., \& Landay, J. A. (2007). Disambiguating speech commands using physical context. In Proceedings of the 9th international conference on Multimodal interfaces (pp. 247-254). New York, NY, USA: ACM. doi:10.1145/1322192.1322235

Figo, D., Diniz, P. C., Ferreira, D. R., \& Cardoso, J. M. (2010). Preprocessing techniques for context recognition from accelerometer data. Personal Ubiquitous Comput., 14(7), 645662. doi:10.1007/s00779-010-0293-9

Freyne, J., Varga, E., Byrne, D., Smeaton, A. F., Smyth, B., \& Jones, G. J. F. (2007). Realising context-sensitive mobile messaging. In Proceedings of the 2007 OTM confederated international conference on On the move to meaningful internet systems - Volume Part I (pp. 407-416). Berlin, Heidelberg: Springer-Verlag. Retrieved from http://dl.acm.org/citation.cfm?id=1780909.1780984

Gunasekera, S. (2012). Android Apps Security (1st ed.). Dordrecht: Springer.

Ho, J., \& Intille, S. S. (2005). Using context-aware computing to reduce the perceived burden of interruptions from mobile devices. In Proceedings of the SIGCHI Conference on Human Factors in Computing Systems (pp. 909-918). New York, NY, USA: ACM. doi:10.1145/1054972.1055100 
Kawaguchi, N., Ogawa, N., Iwasaki, Y., Kaji, K., Terada, T., Murao, K., ... Nishio, N. (2011). HASC Challenge: gathering large scale human activity corpus for the real-world activity understandings. In Proceedings of the 2nd Augmented Human International Conference (pp. 27:1-27:5). New York, NY, USA: ACM. doi:10.1145/1959826.1959853

Ketabdar, H., \& Yüksel, K. A. (2010). Smart ring: controlling call alert functionality based on audio and movement analysis. In Proceedings of the 15th international conference on Intelligent user interfaces (pp. 415-416). New York, NY, USA: ACM. doi:10.1145/1719970.1720049

Khalil, A., \& Connelly, K. (2005a). Context-aware Configuration: A study on improving cell phone awareness. In in the proceedings of Context 05.

Khalil, A., \& Connelly, K. (2005b). Improving cell phone awareness by using calendar information. In Proceedings of the 2005 IFIP TC13 international conference on Human-Computer Interaction (pp. 588-600). Berlin, Heidelberg: Springer-Verlag. doi:10.1007/11555261_48

Khan, A. M. (2011). An empirical study on mobile phone usage. In Proceedings of the 25th BCS Conference on Human-Computer Interaction (pp. 120-125). Swinton, UK, UK: British Computer Society. Retrieved from http://dl.acm.org/citation.cfm?id=2305316.2305339

Kimura, H., Ebisui, J., Funabashi, Y., Yoshii, A., \& Nakajima, T. (2011). iDetective: a persuasive application to motivate healthier behavior using smart phone. In Proceedings of the 2011 ACM Symposium on Applied Computing (pp. 399-404). New York, NY, USA: ACM. doi:10.1145/1982185.1982273

Lemmelä, S., Vetek, A., Mäkelä, K., \& Trendafilov, D. (2008). Designing and evaluating multimodal interaction for mobile contexts. In Proceedings of the 10th international conference on Multimodal interfaces (pp. 265-272). New York, NY, USA: ACM. doi:10.1145/1452392.1452447

Leong, L. H., Kobayashi, S., Koshizuka, N., \& Sakamura, K. (2005). CASIS: a context-aware speech interface system. In Proceedings of the 10th international conference on Intelligent user interfaces (pp. 231-238). New York, NY, USA: ACM. doi:10.1145/1040830.1040880

Leung, K. W.-T., Lee, D. L., \& Lee, W.-C. (2011). CLR: a collaborative location recommendation framework based on co-clustering. In Proceedings of the 34th international ACM SIGIR 
conference on Research and development in Information Retrieval (pp. 305-314). New York, NY, USA: ACM. doi:10.1145/2009916.2009960

Milette, G., \& Stroud, A. (2012). Professional Android Sensor Programming (1st ed.). Hoboken: Wiley.

Miller, C., Blazakis, D., DaiZovi, D., Esser, S., lozzo, V., \& Weinmann, R.-P. (2012). iOS Hacker's Handbook (1st ed.). Hoboken: Wiley.

Miller, C. C. (2013, July 29). Apps That Know What You Want, Before You Do. The New York Times. Retrieved from http://www.nytimes.com/2013/07/30/technology/apps-that-knowwhat-you-want-before-you-do.html

Misra, A., \& Dubey, A. (2013). Android Security: Attacks and Defenses (1st ed.). Hoboken: Taylor and Francis.

Naumann, A. B., Wechsung, I., \& Hurtienne, J. (2010). Multimodal interaction: A suitable strategy for including older users? Interact. Comput., 22(6), 465-474. doi:10.1016/j.intcom.2010.08.005

Nurmi, P., Kukkonen, J., Lagerspetz, E., Suomela, J., \& Floréen, P. (2007). BeTelGeuse: a tool for Bluetooth data gathering. In Proceedings of the ICST 2nd international conference on Body area networks (pp. 21:1-21:8). ICST, Brussels, Belgium, Belgium: ICST (Institute for Computer Sciences, Social-Informatics and Telecommunications Engineering). Retrieved from http://dl.acm.org/citation.cfm?id=1460232.1460253

Ofstad, A., Nicholas, E., Szcodronski, R., \& Choudhury, R. R. (2008). AAMPL: accelerometer augmented mobile phone localization. In Proceedings of the first ACM international workshop on Mobile entity localization and tracking in GPS-less environments (pp. 13-18). New York, NY, USA: ACM. doi:10.1145/1410012.1410016

Ouchi, K., \& Doi, M. (2011). A real-time living activity recognition system using off-the-shelf sensors on a mobile phone. In Proceedings of the 7th international and interdisciplinary conference on Modeling and using context (pp. 226-232). Berlin, Heidelberg: SpringerVerlag. Retrieved from http://dl.acm.org/citation.cfm?id=2045502.2045526 
Ouchi, K., \& Doi, M. (2012). Indoor-outdoor activity recognition by a smartphone. In Proceedings of the 2012 ACM Conference on Ubiquitous Computing (pp. 600-601). New York, NY, USA: ACM. doi:10.1145/2370216.2370324

Pernek, I., Hummel, K. A., \& Kokol, P. (2013). Exercise repetition detection for resistance training based on smartphones. Personal Ubiquitous Comput., 17(4), 771-782. doi:10.1007/s00779-012-0626-y

Rai, P. (2013). Android Application Security Essentials. Birmingham: Packt Publishing.

Sambasivan, N., Ventä, L., Mäntyjärvi, J., Isomursu, M., \& Häkkilä, J. (2009). Designing for social context of mobility: mobile applications for always-on users. In Proceedings of the 21st Annual Conference of the Australian Computer-Human Interaction Special Interest Group: Design: Open 24/7 (pp. 137-144). New York, NY, USA: ACM. doi:10.1145/1738826.1738849

Sayid, R. (2013, May 8). Emotion Sense: App to track your mood could turn your mobile into a pocket therapist. mirror. Retrieved September 24, 2013, from http://www.mirror.co.uk/news/technology-science/technology/emotion-sense-app-trackyour-1874867

Schuster, C., Appeltauer, M., \& Hirschfeld, R. (2011). Context-oriented programming for mobile devices: JCop on Android. In Proceedings of the 3rd International Workshop on ContextOriented Programming (pp. 5:1-5:5). New York, NY, USA: ACM. doi:10.1145/2068736.2068741

Seifert, J., De Luca, A., Conradi, B., \& Hussmann, H. (2010). TreasurePhone: context-sensitive user data protection on mobile phones. In Proceedings of the 8th international conference on Pervasive Computing (pp. 130-137). Berlin, Heidelberg: Springer-Verlag. doi:10.1007/978-3-642-12654-3_8

Siewiorek, D., Smailagic, A., Furukawa, J., Krause, A., Moraveji, N., Reiger, K., ... Wong, F. L. (2003). SenSay: A Context-Aware Mobile Phone. In Proceedings of the 7th IEEE International Symposium on Wearable Computers (p. 248-). Washington, DC, USA: IEEE Computer Society. Retrieved from http://dl.acm.org/citation.cfm?id=946249.946884 
Springer, T., Wustmann, P., Braun, I., Dargie, W., \& Berger, M. (2008). A Comprehensive Approach for Situation-Awareness Based on Sensing and Reasoning about Context. In Proceedings of the 5th international conference on Ubiquitous Intelligence and Computing (pp. 143-157). Berlin, Heidelberg: Springer-Verlag. doi:10.1007/978-3-540-69293-5_13

Thüs, H., Chatti, M. A., Yalcin, E., Pallasch, C., Kyryliuk, B., Mageramov, T., \& Schroeder, U. (2012). Mobile learning in context. Int. J. Technol. Enhanc. Learn., 4(5/6), 332-344. doi:10.1504/IJTEL.2012.051818

Wei, E. J. Y., \& Chan, A. T. S. (2007). Towards context-awareness in ubiquitous computing. In Proceedings of the 2007 international conference on Embedded and ubiquitous computing (pp. 706-717). Berlin, Heidelberg: Springer-Verlag. Retrieved from http://dl.acm.org/citation.cfm?id=1780745.1780822

Westermann, T., \& Möller, S. (2012). MoCCha: a mobile campus app for analyzing user behavior in the field. In Proceedings of the 7th Nordic Conference on Human-Computer Interaction: Making Sense Through Design (pp. 799-800). New York, NY, USA: ACM. doi:10.1145/2399016.2399154

Yamabe, T., Takahashi, K., \& Nakajima, T. (2008). Towards mobility oriented interaction design: experiments in pedestrian navigation on mobile devices. In Proceedings of the 5th Annual International Conference on Mobile and Ubiquitous Systems: Computing, Networking, and Services (pp. 46:1-46:10). ICST, Brussels, Belgium, Belgium: ICST (Institute for Computer Sciences, Social-Informatics and Telecommunications Engineering). doi:10.4108/ICST.MOBIQUITOUS2008.3495

Yu, C.-C., \& Chang, H.-P. (2009). Personalized Location-Based Recommendation Services for Tour Planning in Mobile Tourism Applications. In Proceedings of the 10th International Conference on E-Commerce and Web Technologies (pp. 38-49). Berlin, Heidelberg: Springer-Verlag. doi:10.1007/978-3-642-03964-5_5

Zdziarski, J. (2012). Hacking and Securing iOS Applications: Stealing Data, Hijacking Software, and How to Prevent It (1st ed.). Sebastopol: O'Reilly Media. 